\title{
РАДІОФІЗИКА ГЕОКОСМОСУ
}

DOI: https://doi.org/10.15407/rpra23.04.266

\author{
УДК 537.876 .23 , \\ 550.388 .2 \\ PACS numbers: $94.20 . \mathrm{Vv}$ \\ А. И. РЕЗНИЧЕНКО ${ }^{1,2}$, А. В. КОЛОСКОВ ${ }^{2,3}$, Ю. М. ЯМПОЛЬСКИЙ ${ }^{2}$ \\ ${ }^{1}$ Национальный технический университет "Харьковский политехнический институт”, \\ ул. Кирпичева, 2, г. Харьков, 61002, Украина \\ E-mail: artem.reznychenko@gmail.com \\ ${ }^{2}$ Радиоастрономический институт НАН Украины, \\ ул. Мистецтв, 4, г. Харьков, 61002, Украина \\ E-mail: koloskov@rian.kharkov.ua,yampol@rian.kharkov.ua \\ ${ }^{3}$ Национальный антарктический научный центр МОН Украины, \\ бул. Т. Шевченко, 16, г. Киев, 01601, Украина
}

\section{МОНИТОРИНГ РЕГУЛЯРНЫХ И СПОРАДИЧЕСКИХ ИОНОСФЕРНЫХ ВАРИАЦИЙ НА ОДНОСКАЧКОВЫХ ВЧ РАДИОЛИНИЯХ}

\begin{abstract}
Предмет и цель работы: Диагностика регулярных и спорадических ионосферных вариаций с использованием систематического когерентного мониторинга спектральных характеристик пробных ВЧ сигналов на наклонных односкачковых радиолиниях.

Методы и методология: Регистрация сигналов станции Службы точного времени и частоты проводилась на частоте 9.996 МГи в течение полного 2013 года в двух приемных пунктах - в Низкочастотной обсерватории Радиоастрономического института НАН Украины (с. Мартовое, Харьковской области) и в г. Тромсе (Норвегия). Регистрировались энергетические спектры, по которым оченивались вариации интенсивности и доплеровского смещения частоты (ДСЧ) пробного излучения. Временные ряды информационных характеристик сигналов сопоставлялись с независимыми оценками критических частот ионосферного слоя $F 2$, а также с индексами солнечной и магнитной активности. Установлена их коррелячионная связь.

Результаты: Проведены систематические когерентные измерения вариаций спектральных характеристик пробных ВЧ сигналов на двух односкачковых радиолиниях (высоко- и среднеширотной). В течение полного годового ицкла наблюдений исследованы регулярные (фоновые) вариации суточных зависимостей интенсивностей и ДСЧ пробных сигналов, вызванные изменением условий освещенности контрольных радиотрасс. Установлена эмпирическая зависимость времен характерных изменений ДСЧ и моментов “радиовосхода" и “радиозахода" с прохождением солнечного терминатора на различных высотах среднеширотной и приполярной ионосферы. Для высокоширотной радиотрассы летом определяюшую роль играют условия полярного дня и рост поглощения в полуденное и послеполуденное время. Введен индекс, характеризующий время распространения сигналов на частоте ниже максимально применимой (продолжительность “радиодня”). Для возмущенных ионосферных условий обнаружены 27-дневнье вариации продолжсительности “радиодня” и поведения критических частот ионосферного слоя F2. Периодичность этих вариаций и их синхронизм с индексом солнечной активности F10.7 могут быть объяснены многократным воздействием на ионосферу долгоживущеей активной области в хромосфере при вращении Солнца вокруг своей оси. Возможность использования данных ВЧ мониторинга для диагностики спорадчческих ионосферных возмущений продемонстрирована на примере анализа данных, полученных во время мощчнй рентгеновской вспышки на Солнце и последовавщей за ней магнитной бури.

Заключение: Разработанные методики анализа данных зондирования ионосферы ВЧ сигналами неспециального типа могут быть применены для диагностики и идентификации ионосферных возмущений.
\end{abstract}

Ключевые слова: ионосфера, солнечный терминатор, радиотрасса, солнечная активность, магнитная буря

\section{1. Введение}

В настоящее время существует большой набор методов дистанционного радиозондирования ионосферы. Большинство из них являются активными и предполагают целенаправленное облуче-

(C) А. И. Резниченко, А. В. Колосков, Ю. М. Ямпольский, 2018 ние околоземной плазмы сигналами специального типа. Безусловным достоинством такого подхода является возможность использовать сигналы, форма которых оптимально подобрана для анализа исследуемого типа плазменных образований. С помощью таких классических методов диагностики, как вертикальное зондирование ионосфе- 
ры [1] или метод некогерентного рассеяния [2], получен основной объем наших знаний об ионосфере Земли. Несмотря на очевидные достоинства, активные диагностические методы не свободны от недостатков. Основными из них являются затратность, вызванная необходимостью применения сложных приемопередающих устройств и больших специализированных антенн, высокое энергопотребление, а также дополнительное "загрязнение” окружающего пространства широкополосным зондирующим излучением.

В Радиоастрономическом институте НАН Украины (РИ НАНУ) разработана система диагностики ионосферы ВЧ сигналами широковещательных радиостанций [3]. В ее структуру входит сеть пространственно разнесенных автономных приемных пунктов с дистанционным управлением по сети Интернет. На сегодня приемные пункты успешно функционируют в Украине (Низкочастотная обсерватория - НЧО РИ НАНУ), в Антарктике (Украинская антарктическая станция “Академик Вернадский”), в Северной Скандинавии (г. Тромсе, Норвегия), в экваториальной Африке (Лагос, Нигерия) и в Арктике (о. Свалбард, Шпицберген, Норвегия). Их отличительные особенности: работа в непрерывном мониторинговом режиме, пространственно-временная когерентность, применение серийно выпускаемых цифровых приемников, сопряженных со стандартными персональными компьютерами, удаленный доступ в реальном времени к базам данных по сети Интернет, отображение в режиме онлайн характеристик принятых сигналов на веб-сайте* отдела радиофизики геокосмоса РИ НАНУ. Система в целом и различные ее кластеры использовались во многих задачах радиозондирования природных и стимулированных ионосферных процессов, включая искусственную ионосферную турбулентность, создаваемую мощным ВЧ нагревом [4-6]. Результаты этих исследований послужили основой создания методов многопозиционной доплероскопии и частотно-временной локации ионосферных неоднородностей [7-9].

Настоящая работа посвящена развитию возможностей когерентной ВЧ диагностики среднеширотной и полярной ионосферы с использова-

*URL: www.geospace.com.ua (дата обращения: 07.09.2018). нием систематических двухпозиционных измерений спектральных характеристик сигналов станции Службы точного времени и частоты PBM (Москва, Россия) [10] на двух односкачковых радиолиниях: среднеширотной РВМ-НЧО и высокоширотной РВМ-Тромсе. Объектами исследований являлись регулярные межсезонные и суточные ионосферные вариации, а также ионосферные отклики на мощные геокосмические возмущения.

\section{2. Методика наблюдений и обработки данных}

Измеряемыми характеристиками пробных ВЧ сигналов являлись энергетические спектры, по которым оценивались вариации спектральных плотностей и доплеровские смещения частоты (ДСЧ). Временные зависимости этих величин в свою очередь сопоставлялись с независимыми оценками ионосферных параметров, полученными методом вертикального зондирования ионосферы в пунктах, расположенных вблизи контрольных радиолиний. Непрерывные регистрации пробных сигналов сопровождались мониторингом геомагнитной и анализом солнечной активностей. Основной массив данных измерений проводился на одной из трех рабочих частот станции РВМ 9.996 МГц в течение полного 2013 года. Выбор данной частоты наблюдений был обусловлен выполнением условия преимущественно односкачкового распространения пробных сигналов для обеих радиолиний. Геометрия расположения передатчика и приемных пунктов показана на рис. 1.

Для регистрации пробных сигналов в НЧО и в Тромсе использовались идентичные супергетеродинные приемники WinRadio WR-G313i с цифровым управлением и записью сигналов [11], оснащенные термостатированными кварцевыми задающими генераторами. Это позволяло проводить измерения вариаций ДСЧ с погрешностью не более 0.05 Гц. Точность синхронизации обоих пунктов с всемирным временем UT была не хуже 0.5 мс и обеспечивалась автономными приемниками ГНСС сигналов. В качестве приемных ВЧ антенн использовались малоразмерные рамки и горизонтальные диполи [3]. На выходе аналоговой части приемных устройств пробный сигнал, перенесенный на частоту 500 Гц, оцифровывался 


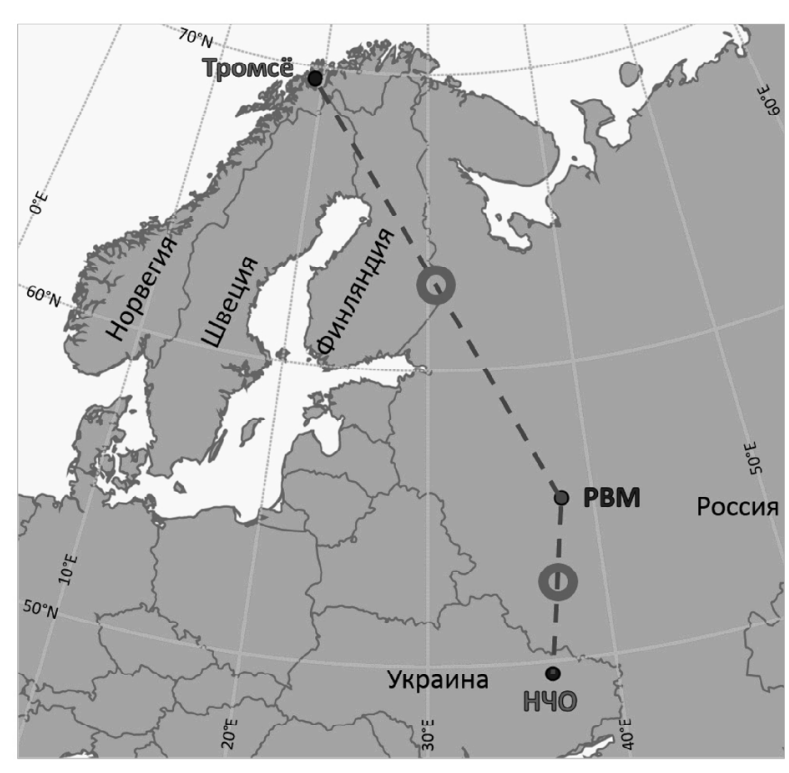

Puc. 1. Геометрия наблюдений: координаты пунктов $55^{\circ} 44^{\prime}$ с. ш., $38^{\circ} 12^{\prime}$ в. Д. (РВM), $49^{\circ} 56^{\prime} 0.5^{\prime \prime}$ с. ш., $36^{\circ} 57^{\prime} 10^{\prime \prime}$ в. Д. (НЧО), 69³9'41" с. Ш., $18^{\circ} 56^{\prime} 30^{\prime \prime}$ в. д. (Тромсе); длины радиотрасс - 650 км (РВМ-НЧО), 1816 км (РВМ-Тромсе); координаты средних точек радиотрасс

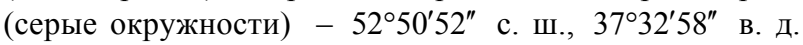

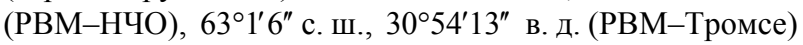

с частотой дискретизации 2 кГц и поступал на регистрирующий персональный компьютер. Выходными данными системы регистрации были энергетические спектры сигналов, определяемые для каждого минутного интервала. Они вычислялись путем усреднения "мгновенных" спектров $S_{p}(f)$, рассчитываемых по фрагментам сигнала длительностью в 20 с. Далее оценивались ДСЧ и интенсивность принятого излучения в полосе частот \pm 1 Гц. В такой же, но смещенной на несколько десятков герц от несущей частоты полосе измерялась интенсивность шума, что позволяло определять соотношение сигнал/шум (С/Ш). При возникновении условий многомодового распространения, и, как следствие, изрезанности спектра частота спектрального максимума вычислялась методом моментов как

$$
f_{\text {дСч }}=\frac{\int_{f_{1}}^{f_{2}} f S_{p}(f) \mathrm{d} f}{\int_{f_{1}}^{f_{2}} S_{p}(f) \mathrm{d} f},
$$

в предположении, что $f_{1}=-5$ Гц, а $f_{2}=+5$ Гц.
Отметим, что данный вид обработки приемлем только для непрерывного режима излучения станции РВМ (475 с в начале каждого получаса). Поэтому при стандартной обработке значения ДСЧ, интенсивности и соотношения С/Ш рассчитывались для первых 8-минутных интервалов в начале каждого получаса и далее усреднялись. Это позволило получить ряды данных с временным разрешением 30 мин (48 точек в сутки) для каждого дня в течение всего 2013 года. Суточные ряды, из которых исключались геомагнитновозмущенные дни, были использованы для расчета усредненных месячных зависимостей. Среднемесячный суточный ход предполагался регулярным и вычитался из текущих суточных рядов. Таким образом определялись вариации ДСЧ, интенсивности и соотношения С/Ш, характеризующие проявления ионосферных возмущений. Возмущенными считались дни, в которые значение глобального $K p$-индекса превышало 3 единицы. Следует отметить, что такая обработка была применима только для “зеркального" односкачкового механизма распространения радиосигналов на контрольных радиотрассах, когда рабочая частота (РЧ) была ниже максимально применимой частоты (МПЧ). В условиях превышения МПЧ интенсивность принятого излучения на обеих трассах резко снижалась (на десятки децибел), спектры существенно расширялись (до десятков герц) и слабая “засветка" приемных пунктов происходила из-за рассеяния сигналов на магнитно-ориентированных ионосферных неоднородностях и возвратно-наклонного распространения [12]. Как правило, переход через уровень максимально применимой частоты [13] происходил два раза в сутки - в утренние и вечерние часы, в соответствии с регулярными градиентами электронной концентрации, когда интенсивность фотоионизации соответственно резко возрастала и уменьшалась. Эти моменты назывались "радиовосходом" и "радиозаходом" сигнала. Момент перехода рабочей частоты через значения МПЧ на трассах PBM-НЧО и РВМ-Тромсе фиксировался по значительному (не менее 20 дБ и 15 дБ соответственно) и резкому (за время менее получаса) изменению интенсивности сигнала. Необходимо отметить, что в моменты времени, когда соотношение С/Ш было около 1, полученные значения интенсивностей и ДСЧ считались недостоверными и в дальнейшем анализе не использовались. 
Очевидно, что продолжительность “зеркальной” засветки приемников определяется как регулярными факторами: сезоном года, местоположением, длиной и ориентацией радиолиний, - так и возмущенностью ионосферы. Для исследования реакции сигналов на ионосферные возмущения был введен индекс, характеризующий вариации продолжительности “зеркальной” засветки (далее - "радиодня”). При его оценивании для каждых суток года определялось время в часах и долях часа, в течение которого интенсивность сигнала не опускалась ниже порогового уровня, отсчитываемого относительно максимума среднемесячной кривой интенсивности. Для трассы РВМ-НЧО порог задавался равным 20 дБ, а для РВМ-Тромсе - 15 дБ. Далее суточные значения, исключая полученные в возмущенные дни, усреднялись за каждый месяц, что давало среднемесячную продолжительность "радиодня", определяемую регулярными факторами. Вариации, связанные с возмущенностью ионосферы, рассчитывались путем центрирования текущих значений относительно среднемесячных зависимостей. Заметим, что в летнее время в средней точке высокоширотной радиотрассы РВМ-Тромсе ионосфера освещена Солнцем в течение всего дня. Поэтому летом в Тромсе падения интенсивности сигнала определялись не переходом РЧ через МПЧ, а ростом поглощения сигнала в дневное время.

Описанные выше ряды вариаций информационных параметров пригодны для выделения и анализа ионосферных возмущений с характерными временными масштабами более получаса. В то же время мониторинг контрольных сигналов РВМ позволяет исследовать и более короткопериодные ионосферные возмущения, например, акустико-гравитационные волны (АГВ). Для их анализа авторами разработаны алгоритмы, позволяющие выделять вариации ДСЧ и интенсивности не только для непрерывных, но и для импульсных режимов работы РВМ. Алгоритм дает возможность получать ряды параметров пробных ВЧ сигналов с разрешением по времени в несколько десятков секунд. Однако, поскольку анализ “быстрых" ионосферных процессов (таких как АГВ) в данной работе не проводился, эта методика будет рассмотрена в отдельной статье.

Для сопоставления информационных характеристик ВЧ сигналов с ионосферными, солнечны- ми и геомагнитными параметрами были использованы синхронные данные других независимых измерений, доступные в сети Интернет:

- значения критической частоты слоя F2 ионосферы - $f o F 2$, измеряемые ионозондами EISCAT [14] (вблизи Тромсе, 69.6 $6^{\circ}$ с. ш., $19.2^{\circ}$ в. д.) и ИЗМИРАН (Москва, 55.47

[15] (временное разрешение данных 15 мин);

- значения локального магнитного $K$-индекса, измеренные в НЧО (временное разрешение данных 3 ч) [16];

- значения локального магнитного $K$-индекса, измеренные в Геофизической обсерватории Тромсе (временное разрешение данных 3 ч) [17];

- значения глобального индекса солнечной активности $F 10.7$ (временное разрешение данных 1 сут) [18].

Все упомянутые выше ряды данных - значения параметров пробных ВЧ сигналов, критических частот, индексов солнечной и магнитной активности - были рассчитаны для каждого месяца 2013 года. Были вычислены также времена восхода и захода Солнца над средними точками радиотрасс на поверхности Земли и высотах 120 и 250 км. Описанию и анализу этих данных посвящены следующие разделы работы.

\section{3. Результаты наблюдений}

\section{Регулярные изменения}

На рис. 2 представлены суточные зависимости интенсивности сигналов для летнего и зимнего сезонов на контрольных радиолиниях. Весной и осенью суточные распределения интенсивности имеют переходной вид между летними и зимними закономерностями и на рисунке не показаны. Вертикальными линиями отмечены времена восхода и захода Солнца, рассчитанные над средними точками контрольных радиотрасс на уровне поверхности Земли и на высотах 120 и 250 км. Характерными моментами прохождения солнечного терминатора для летнего и зимнего сезонов были выбраны дни солнцестояния - 21.06.2013 г. и 21.12.2013 г. Отсутствие суточного хода в ночное время в зимние сезоны связано с невозможностью достоверно определить интенсивность изза низкого соотношения $\mathrm{C} / Ш$.

Усредненный суточный ход ДСЧ сигналов, принятых в НЧО и в Тромсе, для летнего и зимнего сезонов показан на рис. 3 . 

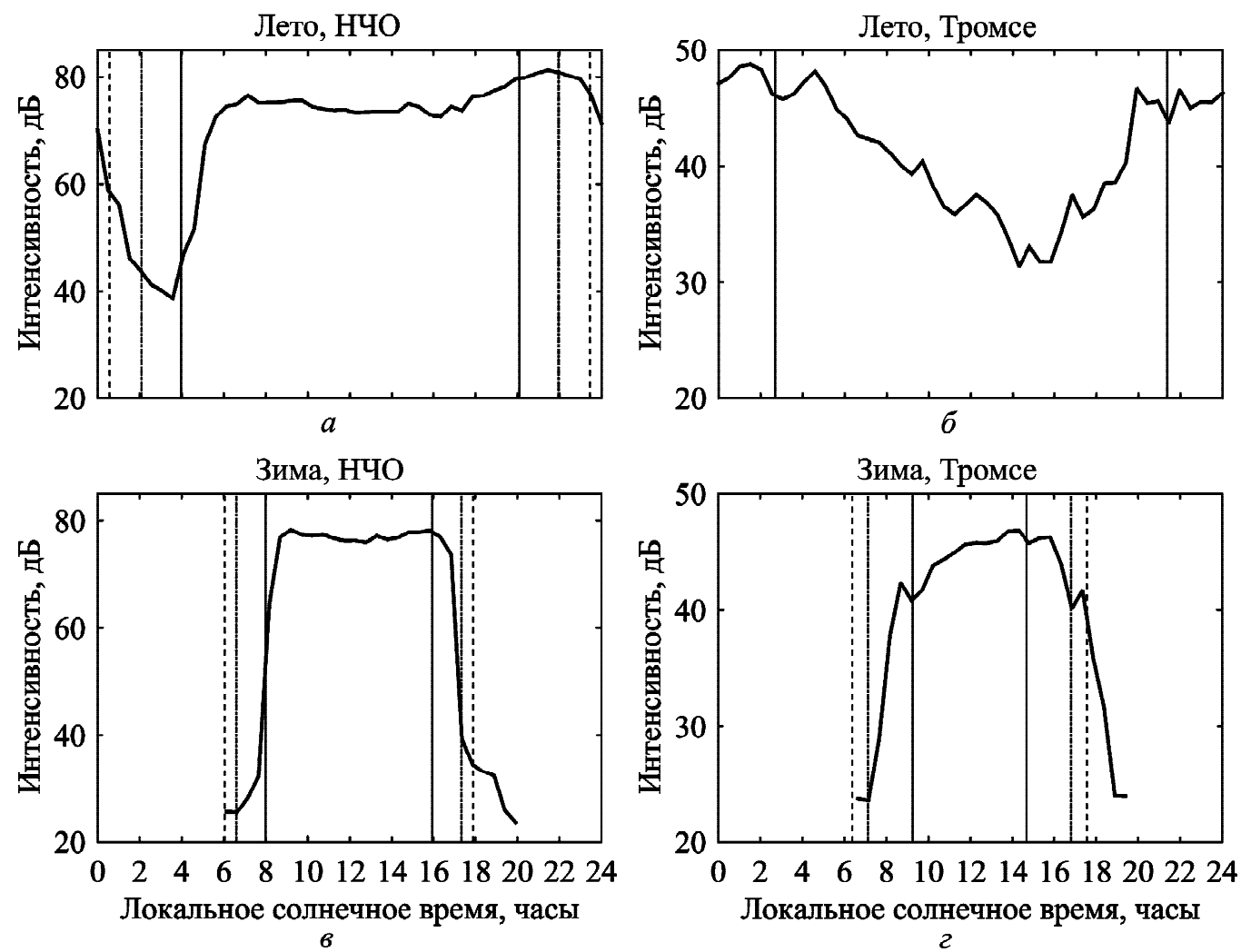

Puc. 2. Суточный ход интенсивности сигнала для летнего (верхние панели) и зимнего (нижние панели) сезонов на среднеширотной $\mathrm{PBM-HЧО}(a, s)$ и высокоширотной РВМ-Тромсе $(\sigma, 2)$ радиотрассах. Вертикальные линии соответствуют усредненным временам восхода и захода Солнца над средними точками радиотрасс на уровне поверхности Земли (сплошные линии) и на высотах 120 км (штрих-пунктирные линии), 250 км (пунктирные линии)

Динамические суточно-годовые зависимости ДСЧ сигнала для обеих радиотрасс показаны на рис. 4. По вертикальной оси отложено время суток (слева - локальное солнечное время в средней точке радиотрассы, справа - мировое время UT), на горизонтальной оси указан номер дня, отсчитанный от начала года. Значения ДСЧ в герцах отображены в условных цветах. Белые участки соответствуют моментам, когда соотношение C/Ш было недостаточным для надежного определения ДСЧ сигнала и моментам отсутствия данных.

Суточно-годовые зависимости интенсивностей сигнала в НЧО и в Тромсе показаны на рис. $5, a$ и 5,6 . Цветом отображены значения интенсивности сигнала в децибелах. Белые участки соответствуют моментам отсутствия данных.

Рис. 6, $a$ отображает вариации продолжительности "радиодня" относительно среднемесячных значений для радиолиний $\mathrm{PBM}-\mathrm{HЧO}$ и $\mathrm{PBM}-$ Тромсе в течение всего 2013 года. На рис. 6, б показаны изменения в тот же период времени индекса $F 10.7$, характеризующего солнечную активность, и характеристики ионосферы - критической частоты $f o F 2$, представленной суточными значениями, усредненными за время "радиодня".

\section{Возмущценные условия}

Систематические измерения информационных параметров и восстановление их регулярных (среднесуточных и сезонных) зависимостей позволяют выделить периоды аномального поведения пробных ВЧ сигналов, стимулированных мощными геофизическими возмущениями. В качестве примера далее проанализированы отклики параметров сигналов на контрольных радиолиниях на рентгеновскую вспышку на Солнце и последовавшую за ней геомагнитную бурю 17-18 марта 2013 г.

На рис. 7 изображены вариации интенсивности сигнала в НЧО и в Тромсе, а возмущенность условий распространения проиллюстрирована изменениями потока солнечного рентгеновского 

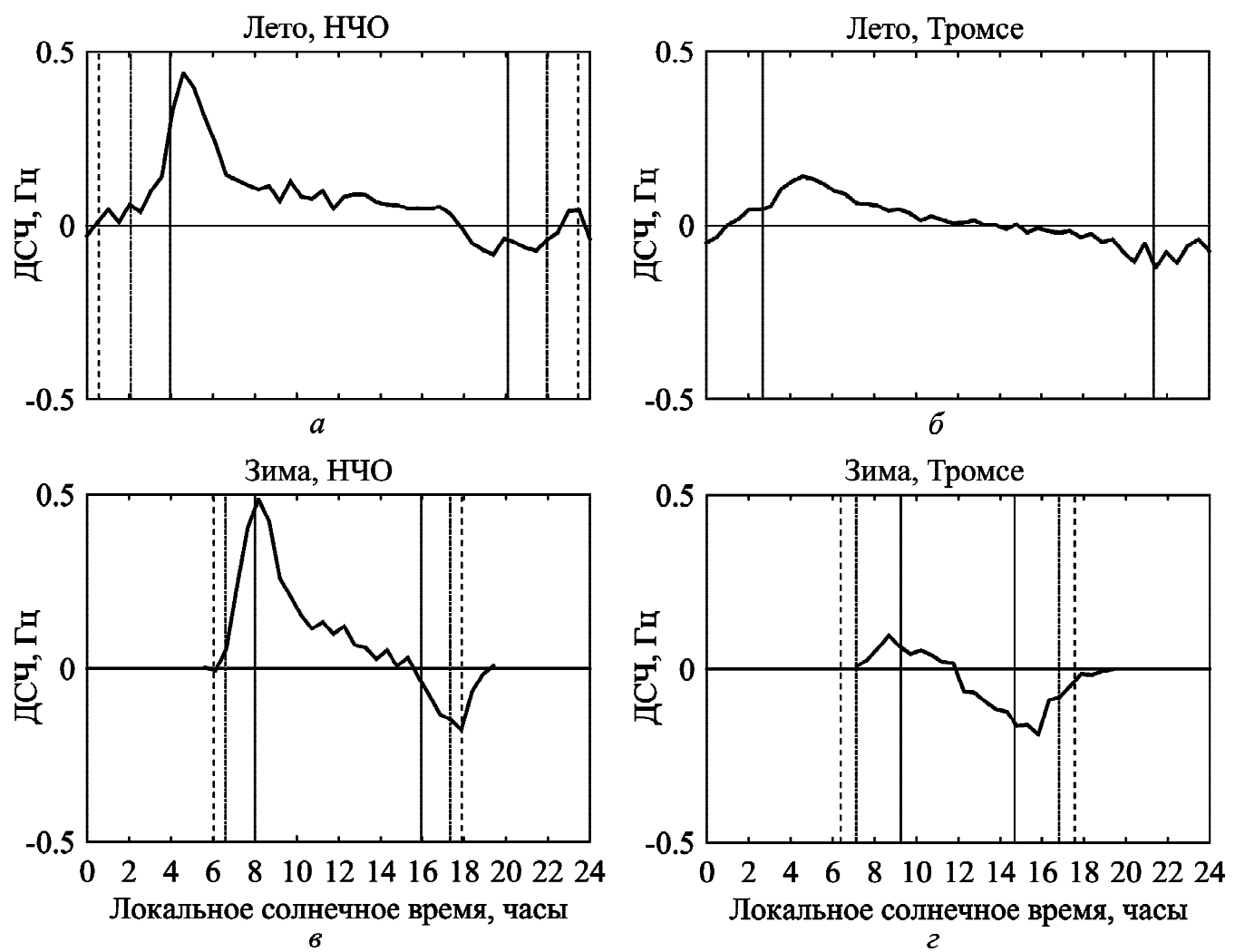

Puc. 3. Суточный ход ДСЧ сигнала для летнего (верхние панели) и зимнего (нижние панели) сезонов на среднеширотной PBM-НЧО $(a, b)$ и высокоширотной РВМ-Тромсе $(\sigma, 2)$ радиотрассах. Вертикальные линии соответствуют усредненным временам восхода и захода Солнца над средними точками радиотрасс на уровне поверхности Земли (сплошные линии) и для высот 120 км (штрих-пунктирные линии), 250 км (пунктирные линии)

излучения и геомагнитных индексов в течение четырех суток. Фоновые вариации интенсивности сигнала (пунктирные линии) получены в результате усреднения суточных зависимостей за весь месяц март 2013 г.

\section{4. Обсуждение результатов наблюдений}

Переходя к обсуждению результатов наблюдений, прокомментируем суточные изменения интенсивности сигнала летом и зимой, показанные на рис. 2. Для обоих сезонов перепад между ночными и дневными значениями интенсивности на трассе РВМ-НЧО больше, чем на радиолинии РВМ-Тромсе. Из рисунка видно, что зимой перепады между дневной и ночной интенсивностями составляют около 50 дБ в НЧО и около 20 дБ в Тромсе. В НЧО (рис. 2, в) суточный ход интенсивности хорошо соответствует временам восхода Солнца на уровне поверхности Земли и моментам захода на высоте 120 км. На высокоширотной трассе (рис. 2,2 ) характерным является увеличение интенсивности сигнала после восхода Солнца на ионосферных высотах в области Е и уменьшение интенсивности после прохождения вечернего терминатора на высотах F области (250 км).

Для летнего сезона в НЧО (рис. 2, a) наблюдается запаздывание роста интенсивности сигнала относительно момента прохождения утреннего терминатора по поверхности Земли и сохранение высокой интенсивности сигнала после захода Солнца на высотах F области. Суточно-годовые зависимости интенсивности (рис. 5) показывают, что такое поведение сохраняется на протяжении весеннего, летнего и осеннего сезонов. Интересной особенностью поведения интенсивности в летнее время является также послеполуденный минимум. Он связан в основном с поглощением в нижней ионосфере в это время. Данный эффект лучше всего проявляется с 140-го по 250-й день года (см. рис. 5).

Для Тромсе летом (рис. 2, б) наблюдается обратная зависимость поведения интенсивности пробного сигнала от времени суток с максиму- 

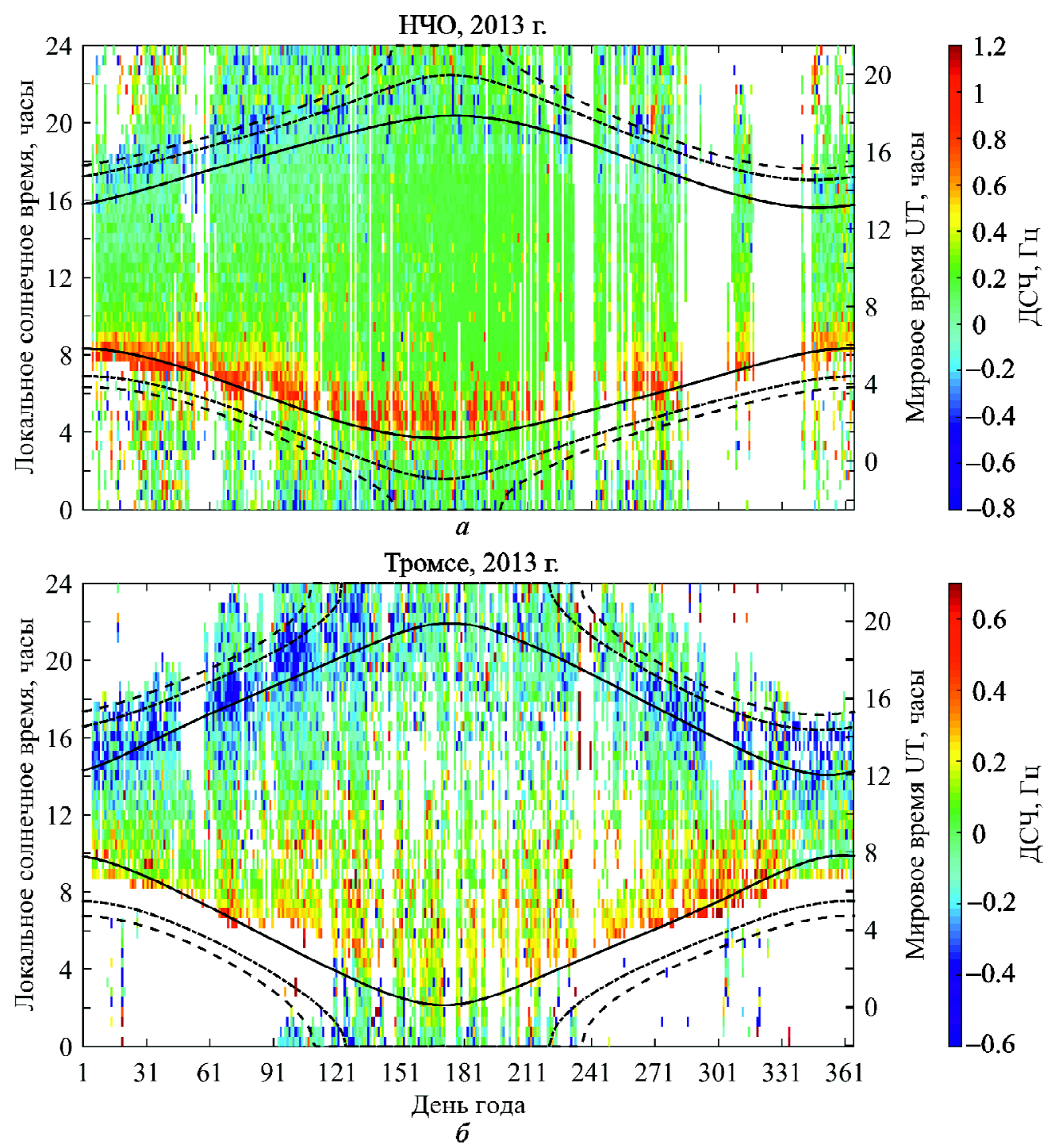

Puc. 4. Суточно-годовая зависимость ДСЧ сигнала на среднеширотной РВМ-НЧО ( $a$ ) и высокоширотной РВМ-Тромсе (б) радиотрассах. Черные линии соответствуют времени прохождения утреннего (внизу на панелях) и вечернего (вверху на панелях) солнечного терминатора через средние точки радиотрасс на уровне поверхности Земли (сплошные линии) и на высотах 120 км (штрих-пунктирные линии) и 250 км (пунктирные линии)

мом ночью и минимумом днем. Такое поведение интенсивности определяют условия полярного дня, когда области Е и $\mathrm{F}$ ионосферы в середине трассы постоянно освещены Солнцем. Вследствие этого РЧ летом в течение всех суток остаются ниже МПЧ, а дневное уменьшение интенсивности, составляющее в среднем 15 дБ, определяется в основном поглощением в нижней ионосфере [19]. Несмотря на то, что обратная зависимость изменений интенсивности в Тромсе наблюдается только летом, ее значительное дневное уменьшение, как это видно из рис. 5 , регистрируется более продолжительное время - с начала весны и до середины осени. Поэтому весной и в первую половину осени интенсивность имеет два минимума: ночной, связанный с “радиозаходом" и дневной, вызванный ростом поглощения.

Рис. 3 показывает, что регулярное поведение ДСЧ характеризуется положительным подъемом после восхода Солнца на высотах Е области ионосферы и отрицательным смещением вблизи захода. Знаки ДСЧ соответствуют уменьшению действующей высоты отражения сигнала в утренние часы и ее росту вечером. Более слабые суточные вариации ДСЧ на высокоширотной трассе летом объясняются меньшим контрастом меж- 

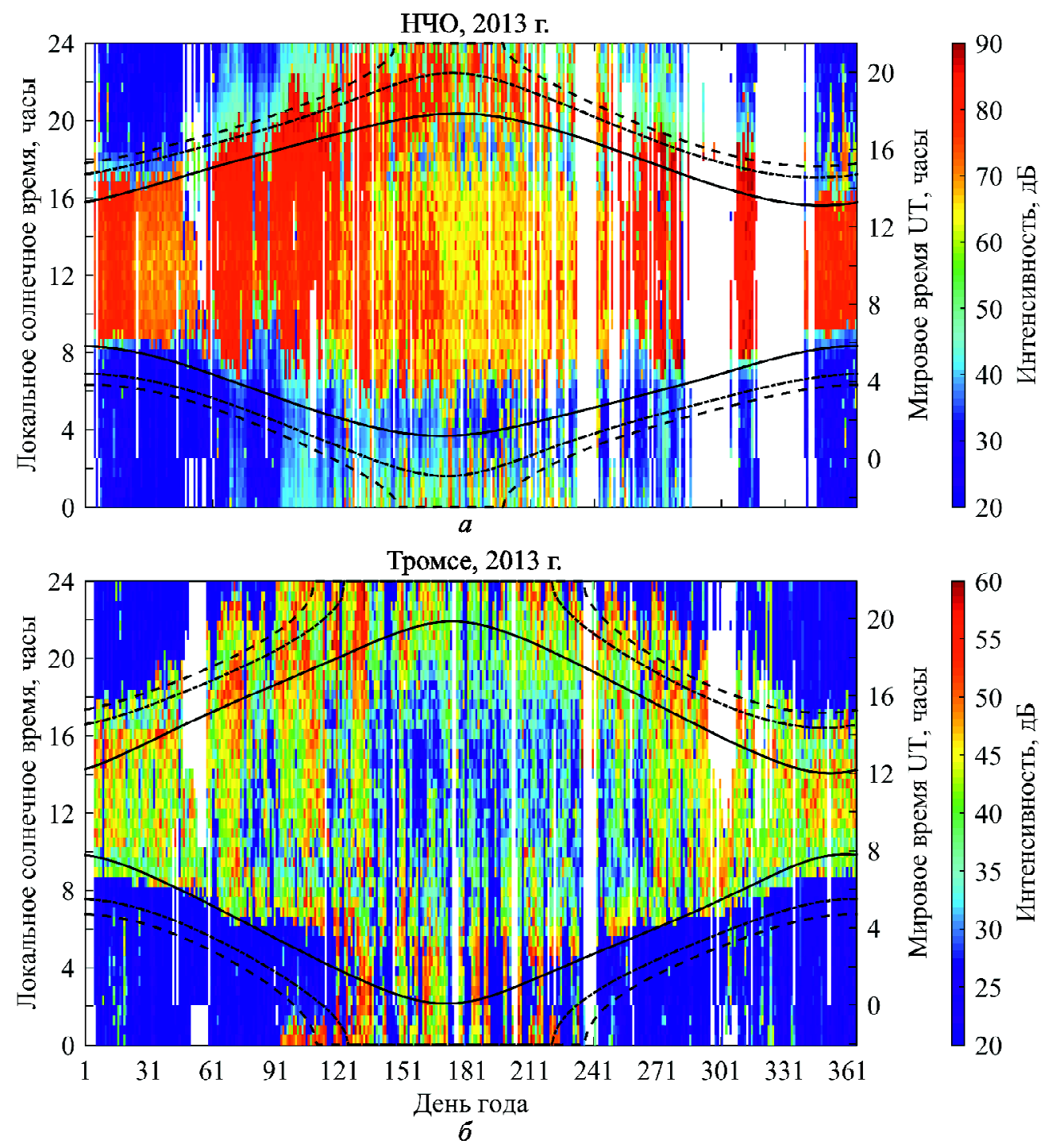

Puc. 5. Суточно-годовая зависимость интенсивности сигнала на среднеширотной РВМ-НЧО $(a)$ и высокоширотной PBM-Тромсе (б) радиотрассах. Черные линии соответствуют времени прохождения утреннего (внизу на панелях) и вечернего (вверху на панелях) солнечного терминатора через средние точки радиотрасс на высотах 0 км (сплошные линии), 120 км (штрих-пунктирые линии) и 250 км (пунктирые линии) над поверхностью Земли

ду “ночными” и “дневными” условиями освещенности. Как уже отмечалось, на широтах выше $60^{\circ}$ летом на высотах отражения в середине радиотрассы РВМ-Тромсе Солнце не заходит за горизонт и освещение носит переходной сумеречный характер. Для летнего и зимнего сезонов в НЧО (рис. $3, a, b$ ) максимальные значения ДСЧ фиксируются непосредственно после прохождения по поверхности Земли линии утреннего терминатора, а минимальные - после вечернего. Для высокоширотной трассы (рис. 3, 6, г) картина более сложная, учитывая ее большую протяженность и близость к полярному овалу. Летом на- блюдается значительное запаздывание положительного пика ДСЧ относительно восхода Солнца на уровне поверхности Земли, а зимой положительный пик ДСЧ наблюдается до прохождения утреннего терминатора на нулевой высоте.

Отмеченные выше особенности можно более подробно проследить на суточно-годовых зависимостях ДСЧ, построенных для обоих наблюдательных пунктов (рис. 4). В обоих случаях утренний положительный максимум ДСЧ совпадает по времени с прохождением утреннего терминатора по поверхности Земли только зимой и запаздывает относительно него в другие сезоны. 

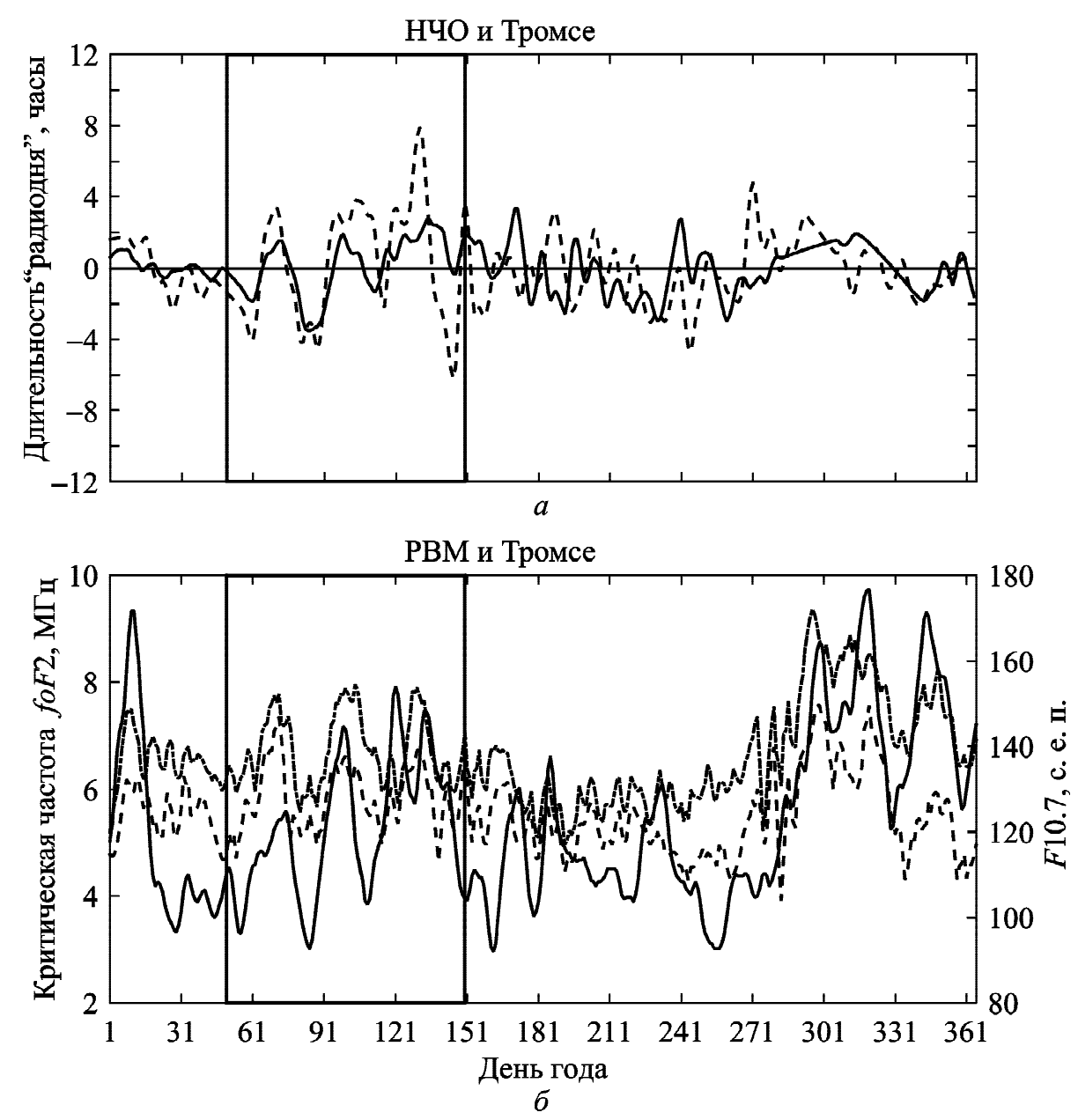

Puc. 6. Вариации продолжительности “радиодня" на протяжении 2013 года на радиотрассах РВМ-НЧО (сплошная кривая) и РВМ-Тромсе (пунктирная кривая) (a); месячные изменения индекса солнечной активности $F 10.7$ $\left(1\right.$ с. е. $\Pi=10^{-22}$ Вт $/\left(\mathrm{M}^{2} \cdot\right.$ Гц )) (сплошная кривая), средних за освещенный период суток значений частоты $f o F 2$ ионосферного слоя F2 над РВМ (штрих-пунктирная кривая) и над Тромсе (пунктирная кривая) (б)

Отрицательный вечерний минимум ДСЧ соответствует интервалу между временами прохождения солнечного терминатора по поверхности и на ионосферных высотах.

Кроме регулярных сезонных закономерностей поведения параметров ВЧ сигналов, определяемых изменением условий освещенности, суточно-годовые зависимости (рис. 4 и рис. 5) позволяют отслеживать “медленные" квазипериодические вариации параметров пробного излучения. Так, на рис. 4, $б$ с 50-го по 150-й день можно проследить три минимума амплитуды вечернего отрицательного пика ДСЧ в Тромсе, отстоящие друг от друга на $25 \div 30$ дней. Положения этих минимумов ДСЧ соответствуют максимумам продолжительности “радиодня", которые лучше всего заметны на суточно-годовых зависимос- тях интенсивности в НЧО (рис. 5, $a$ ). Для изучения данного эффекта были проанализированы вариации продолжительности “радиодня” (рис. 6, $a$ ) совместно с индексом солнечной активности $F 10.7$ и средними за освещенный период суток значениями критических частот в Тромсе и вблизи РВМ (рис. 6, б). Как видно из рисунка, с 50-го по 150-й день (между вертикальными линиями на рис. 6) параметры ВЧ сигналов, индексы солнечной активности и критические частоты демонстрируют сходные квазипериодические вариации. Построение взаимных спектров позволило уточнить период вариаций, который в среднем составил 27.8 сут. Данное значение соответствует периоду вращения Солнца вокруг своей оси. Это позволяет предположить, что активная область на Солнце, которая стала источ- 

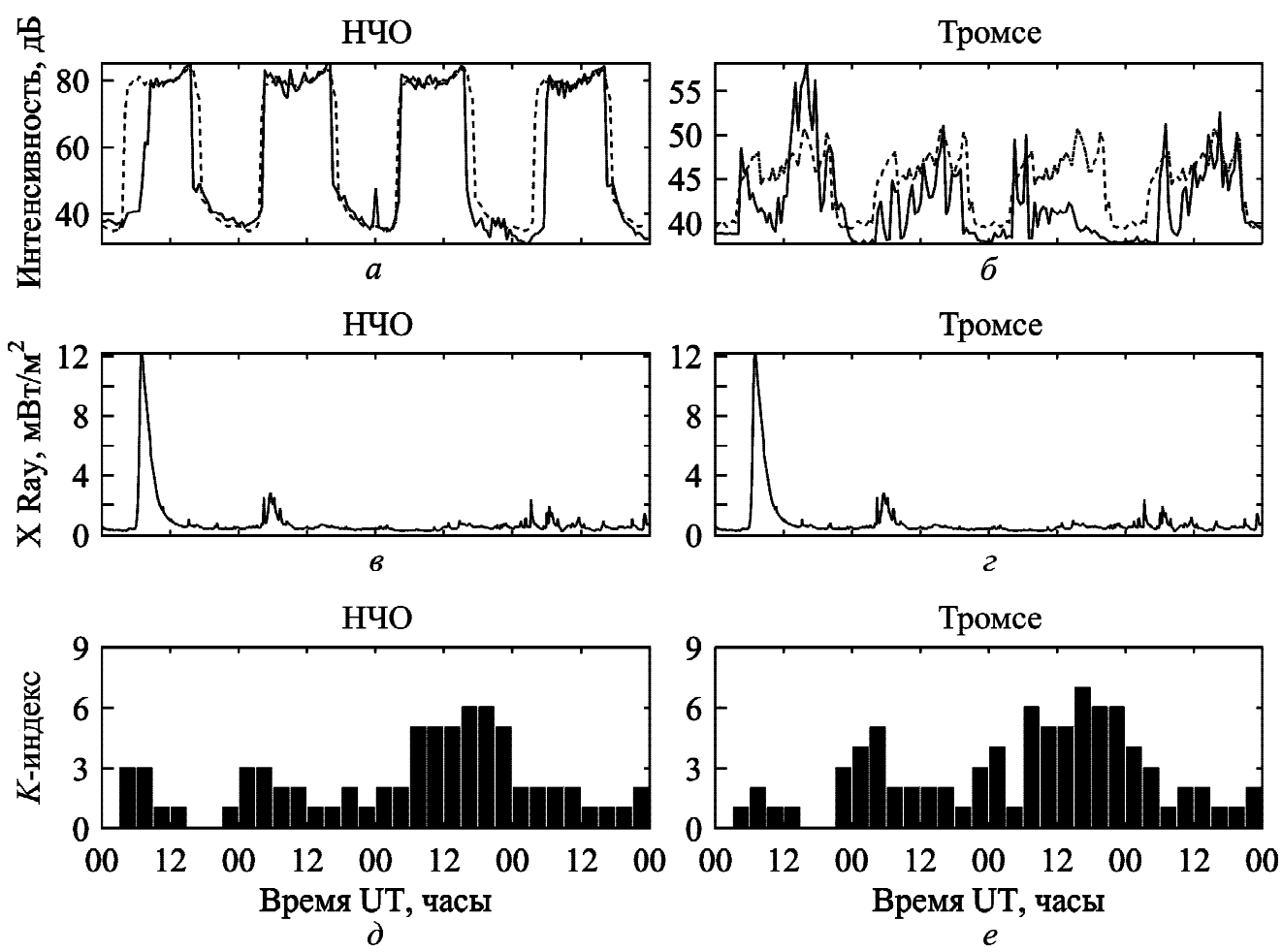

Puc. 7. Вариации интенсивности принятого ВЧ сигнала (сплошные линии) и фоновой интенсивности ВЧ сигнала (пунктирные линии) в НЧО (a) и в Тромсе (б), потока солнечного рентгеновского излучения в полосе $1 \div 8 \AA$ в НЧО (в) и в Тромсе (2), локальных геомагнитных $K$-индексов в НЧО (d) и в Тромсе $(e)$ 15-18 марта 2013 г.

ником рентгеновской вспышки в 74-й день года (см. рис. 7, в, г), продолжала существовать на протяжении нескольких “солнечных суток”. Ее излучение при каждом полном обороте Солнца вокруг оси модулировало индекс солнечной активности, критические частоты ионосферы и соответственно условия распространения ВЧ сигналов на контрольных радиолиниях.

Для подтверждения этого предположения для первого полугодия 2013 года, когда вариации проявлялись наиболее явно, были рассчитаны коэффициенты корреляции между индексом F10.7, критическими частотами ионосферы и вариациями продолжительности “радиодня". Для критической частоты, измеренной вблизи РВМ, коэффициент корреляции с индексом $F 10.7$ составил 0.62 , а для критической частоты, измеренной в Тромсе, -0.61 . Значения коэффициента корреляции индекса $F 10.7$ с вариациями продолжительности "радиодня" оказались того же порядка и составили 0.72 для НЧО и 0.60 для Тромсе. Таким образом, индекс продолжительности “радиодня”, который сравнительно несложно рассчитать по параметрам пробных ВЧ сигналов на конт- рольных радиолиниях, проявил себя таким же эффективным индикатором вариаций спорадической солнечной активности, как и критические частоты ионосферы, мониторинг которых является более сложной и затратной задачей.

Анализ параметров ВЧ сигналов на контрольных радиолиниях позволяет изучать не только описанные выше "медленные" ионосферные возмущения с периодами в десятки дней, но и более быстрые процессы. Рис. 7 демонстрирует реакцию параметров ВЧ сигналов на возмущения радиационного и корпускулярного потоков солнечной радиации. Из рисунка видно, что рентгеновская вспышка класса M1.1, продолжавшаяся с 5:46 до 8:35 UT 15 марта 2013г., вызвала уменьшение продолжительности “радиодня" в обоих пунктах наблюдения. Наиболее явно эффект проявился на среднеширотной радиолинии. Для высокоширотной, более длинной, радиотрассы уменьшение интенсивности было менее значимо. Как показывают данные спутниковых измерений [20], солнечная вспышка сопровождалась выбросом корональной массы, вызвавшим магнитную бурю класса G2 17-18 марта 2013 г. 
Как видно из рис. 7, интенсивность пробного ВЧ излучения отреагировала на это событие только на приполярной радиотрассе, в то время как на среднеширотной реакция отсутствовала. Это объясняется близостью трассы РВМ-Тромсе к овалу полярных сияний и проявлению эффектов высыпания энергичных частиц и роста аврорального поглощения [19] во время магнитной бури. Приведенные примеры демонстрируют возможность идентификации ионосферных возмущений разных типов без использования традиционных активных методов зондирования ионосферы.

\section{5. Выводы}

1. Проанализированы годовые данные непрерывных систематических наблюдений спектральных и энергетических характеристик пробных ВЧ радиосигналов на двух односкачковых радиолиниях в средних и высоких широтах.

2. Установлены суточно-сезонные зависимости поведения интенсивности и доплеровского смещеня частоты сигналов, вызванные регулярными изменениями освещенности контрольных радиотрасс.

3. Установлена эмпирическая связь времен "радиовосхода" и "радиозахода" с временем прохождения солнечного терминатора на различных высотах для среднеширотной и приполярной ионосферы.

4. Введен индекс продолжительности "радиодня", характеризующий время распространения сигналов на рабочей частоте ниже максимально применимой. С его помощью обнаружена 27-дневная периодичность, связанная с вариацией солнечной активности, проявляющаяся также в поведении критических частот $f o F 2$ на контрольных радиолиниях.

5. Проанализированы вариации интенсивности сигналов во время мощной рентгеновской вспышки на Солнце и последующей за ней магнитной бури, которая сопровождалась ионосферной бурей. Сопоставление информационных параметров сигналов с независимыми данными измерений потока солнечного рентгеновского излучения и индекса магнитной активности показало их хорошее соответствие.

6. Продемонстрирована возможность диагностики и идентификации возмущений ионосферы с помощью систематического когерентного зондирования на односкачковых ВЧ радиолиниях с использованием излучения широковещательных радиостанций.

Выполненные исследования носят эмпирический наблюдательный характер и, по мнению авторов, представляют интерес для последующего физического моделирования.

Работа выполнена в рамках исследований по ведомственным НИР НАН Украины "Ятаган-3" (номер госрегистрации 0116U000035) и “Віддзеркалення" (0118U100207), а также частично финансово поддержана конкурсными НИР "Шпицберген-2018” (0118U000562) и “Гелиомакс-2018” (0118U100280).

Авторы считают своим приятным долгом поблагодарить коллег из Арктического университета г. Тромсе (Норвегия) и сотрудников Низкочастотной обсерватории РИ НАН Украины за содействие в проведении непрерывных многолетних измерений ВЧ сигналов на контрольных радиолиниях. Мы признательны также коллегам из отдела радиофизики геокосмоса А. В. Зализовскому, С. Б. Кащееву, А. А. Сопину и А. С. Кащееву за помощь в обработке данных и полезные обсуждения результатов.

\section{СПИСОК ЛИТЕРАТУРЫ}

1. Bibl K. and Reinisch B. W. The Universal Digital Ionosonde. Radio Sci. 1978. Vol. 13, No 3. P. 519-530. DOI: 10.1029/rs013i003p00519

2. Evans J. V. Theory and practice of ionosphere study by Thomson scatter radar. Proc. IEEE. 1969. Vol. 57, No. 4. P. 496-530. DOI: 10.1109/PROC.1969.7005

3. Колосков А. В., Ямпольский Ю. М., Зализовский А. В., Галушко В. Г., Кащеев А. С., Ла Хоз С., Брекке А., Белей В. С., Ритвелд М. Т. Сеть Интернет-управляемых ВЧ приемников для ионосферных исследований. Padioфізика і радіоастрономія. 2014. Т. 19, № 4. С. 324-335. DOI: $10.15407 /$ rpra19.04.324

4. Zalizovski A. V., Galushko V. G., Kashcheev A. S., Koloskov A. V., Yampolski Yu. M., Egorov I. B., and Popov A. V. Doppler Selection of HF Radiosignals on Long Paths. Geomagn. Aeron. 2007. Vol. 47, No. 5. P. 636-646. DOI: $10.1134 / \mathrm{S} 001679320705012 \mathrm{X}$

5. Zalizovski A. V., Kascheev S. B., Yampolski Y. M., Galushko V. G., Beley V. S., Isham B., Rietveld M. T., La Hoz C., Brekke A., Blagoveshchenskaya N. F., and Kornienko V. A. Self-scattering of a powerful HF radio wave on stimulated ionospheric turbulence. Radio Sci. 2009. Vol. 44, No 3. id. RS3010. DOI: 10.1029/2008RS004111 6. Najmi A., Milikh G., Yampolski Y. M., Koloskov A. V., Sopin A. A., Zalizovski A. V., Bernhardt P, Briczinski S., Siefring C., Chiang K., Morton Y., Taylor S., Mahmoudian A., Bristow W., Ruohoniemi M., and Papadopoulos K. Studies of the ionospheric turbulence excited by the fourth gyroharmonic at HAARP. J. Geophys. Res. Space 
Phys. 2015. Vol. 120, No. 8. P. 6646-6660. DOI: 10.1002/ 2015JA021341

7. Кащеев С. Б., Колосков А. В., Зализовский А. В., Галушко В. Г., Пикулик И. И., Ямпольский Ю. М., Куркин В. И., Литовкин Г. И., Орлов А. И., Петько П. В. Экспериментальные исследования спектральных характеристик КВ сигналов на дальних и сверхдальних трассах. Радіофізика і радіоастрономія. 2009. Т. 14, No. 1. C. 12-25. URL: http://rpra-journal.org.ua/index. $\mathrm{php} / \mathrm{ra} / \mathrm{article} / \mathrm{view} / 529$ (дата обращения: 07.09.2018).

8. Кащеев С. Б., Зализовский А. В., Колосков А. В., Галушко В. Г., Пикулик И. И., Ямпольский Ю. М., Куркин В. И., Литовкин Г. И., Орлов А. И. Вариации частоты КВ сигналов на протяженных трассах во время солнечного затмения. Радіофізика і радіоастрономія. 2009. T. 14, No. 4. C. 353-366. URL: http://rpra-journal. org.ua/index.php/ra/article/view/500 (дата обращения: 07.09.2018).

9. Зализовский А. В., Колосков А. В., Ямпольский Ю. М. Исследования в Антарктике частотно-временных характеристик ВЧ сигналов на сверхдальних радиолиниях, Украйнський антарктичний журнал. 2015. No. 14. C. 124-137. URL: http://nbuv.gov.ua/UJRN/uazh_2015_14_14 (дата обращения: 07.09.2018).

10. Эталонные сигналы частоты и времени. Характеристики и программы передач через радиостанции, телевидение и сеть звукового вещания. Бюллетень. Под ред. Г. Т. Черенкова. Москва: Издательство стандартов, 1979. $27 \mathrm{c}$.

11. WR-G313i. WINRADIO by RADIXON. Last updated: 2018. URL: https://www.winradio.com/home/g313i.htm (дата обращения: 07.09.2018).

12. Galushko V. G. and Yampolski Yu. M. Ionospheric diagnostics using wave field diffraction near the caustic. Radio Sci. 1996. Vol. 31, No. 5. P. 1109-1118. DOI: 10.1029/ 96RS01737

13. Долуханов М. П. Распространение радиоволн. Москва: Связь, 1972. 440 с.

14. Archive ionograms Tromsø 2003 -> today. Tromsø Geophysical Observatory. UiT, The Arctic University of Norway. Last updated: 30.01.2015. URL: http://geo.phys. uit.no/ionodata/ionosonde.html (дата обращения: 07.09.2018).

15. Moscow Digisonde DPS-4 \#045. IZMIRAN. Last updated: 2006. URL: http://dps.izmiran.ru (дата обращения: 07.09.2018).

16. K-Indexes of geomagnetic activity. IRA NASU, Department of Radiophysics of Geospace. Last updated: 2018. URL: http://geospace.com.ua/en/data/metmag_ki.php (дата обращения: 07.09.2018).

17. Geomagnetic Data. Tromsø Geophysical Observatory. UiT, The Arctic University of Norway. Last updated: 16.04.2018. URL: http://flux.phys.uit.no/geomag.html (дата обращения: 07.09.2018).

18. Data Service. Space weather prediction center, National oceanic and atmospheric administration. Last updated: 2018. URL: ftp://ftp.swpc.noaa.gov/pub/warehouse/2013/ (дата обращения: 07.09.2018).

19. Hunsucker R. D and Hargreaves J. K. The High-Latitude Ionosphere and its Effects on Radio Propagation. Cambridge: Cambridge University Press, 2002. DOI: 10.1017/ CBO9780511535758
20. Solar Storm Near Earth Caused by March 15, 2013 Fast CME. Goddard Media Studios. Last updated: 18.03.2018. URL: https://svs.gsfc.nasa.gov/11225 (дата обращения: 07.09.2018).

\section{REFERENCES}

1. BIBL, K. and REINISCH, B. W., 1978. The Universal Digital Ionosonde. Radio Sci. vol. 13, no. 3, pp. 519-530. DOI: $10.1029 / \mathrm{rs} 013 \mathrm{i} 003 \mathrm{p} 00519$

2. EVANS, J. V., 1969. Theory and practice of ionosphere study by Thomson scatter radar. Proc. IEEE. vol. 57, no. 4, pp. 496-530. DOI: 10.1109/PROC.1969.7005

3. KOLOSKOV, A. V., YAMPOLSKI, Y. M., ZALIZOVSKY, A. V., GALUSHKO, V. G., KASHCHEYEV, A. C., LA HOZ, C., BREKKE, A., BELYEY, V. S. and RIETVELD, M. T., 2014. Network of internet-controlled HF receivers for ionospheric researches. Radio Phys. Radio Astron. vol. 19, no. 4, pp. 324-335. (in Russian). DOI: 10.15407/rpra19.04.324

4. ZALIZOVSKI, A. V., GALUSHKO, V. G., KASHCHEEV, A. S., KOLOSKOV, A. V., YAMPOLSKI, Y. M., EGOROV, I. B. and POPOV, A. V., 2007. Doppler Selection of HF Radiosignals on Long Paths. Geomagn. Aeron. vol. 47, no. 5, pp. 636-646. DOI: 10.1134/S001679320705012X

5. ZALIZOVSKI, A. V., KASCHEEV, S. B., YAMPOLSKI, Y. M., GALUSHKO, V. G., BELEY, V. S., ISHAM, B., RIETVELD, M. T., LA HOZ, C., BREKKE, A., BLAGOVESHCHENSKAYA, N. F. and KORNIENKO, V. A., 2009. Self-scattering of a powerful HF radio wave on stimulated ionospheric turbulence. Radio Sci. vol. 44, no. 3, id. RS3010. DOI: 10.1029/2008RS004111

6. NAJMI, A., MILIKH, G., YAMPOLSKI, Y. M., KOLOSKOV, A. V., SOPIN, A. A., ZALIZOVSKI, A. V., BERNHARDT, P., BRICZINSKI, S., SIEFRING, C., CHIANG, K., MORTON, Y., TAYLOR, S., MAHMOUDIAN, A., BRISTOW, W., RUOHONIEMI, M. and PAPADOPOULOS, K., 2015. Studies of the ionospheric turbulence excited by the fourth gyroharmonic at HAARP. J. Geophys. Res. Space Phys. vol. 120, no. 8, pp. 6646-6660. DOI: $10.1002 / 2015 J A 021341$

7. KASCHEEV, S. B., KOLOSKOV, A. V., ZALIZOVSKI, A. V., GALUSHKO, V. G., PIKULIK, I. I., YAMPOLSKI, Y. M., KURKIN, V. I., LITOVKIN, G. I., ORLOV, A. I. and PETKO, P. V., 2009. Experimental Investigation of Spectral Characteristics of HF Signals at Long and Ultra-Long-Range Radio Paths. Radio Phys. Radio Astron. [online]. vol. 14, no. 1, pp. 12-25. (in Russian). [viewed: 07.09.2018]. Available from: http://rpra-journal. org.ua/index.php/ra/article/view/529

8. KASCHEEV, S. B., ZALIZOVSKI, A. V., KOLOSKOV, A. V., GALUSHKO, V. G., PIKULIK, I. I., YAMPOLSKI, Y. M., KURKIN, V. I., LITOVKIN, G. I. and ORLOV, A. I., 2009. Frequency Variations of HF Signals at Long-Range Radio Paths during a Solar Eclipse. Radio Phys. Radio Astron. [online]. vol. 4, no. 4, pp. 353-366 (in Russian). [viewed: 07.09.2018]. Available from: http://rpra-journal.org.ua/index.php/ra/article/view/500

9. ZALIZOVSKI, A. V., KOLOSKOV, A. V. and YAMPOLSKI, Y. M., 2015. Studying in Antarctica the time-frequency characteristics of HF signals at the long radio paths. Ukrainian Antarctic Journal [online]. no. 14, pp. 124-137. 
(in Russian). [viewed: 07.09.2018]. Available from: http://nbuv.gov.ua/UJRN/uazh 2015 1414

10. CHERENKOV, G. T., ed. 1979. Standard frequency and time signals. Characteristics and schedule of transmittings through radio stations, television and sound broadcast network. The Bulletin. Moscow, USSR: Standards Publishing House. (in Russian).

11. WINRADIO by RADIXON, 2018. WR-G313i [online]. [viewed 07.09.2018]. Available from: https://www.winradio. com/home/g313i.htm

12. GALUSHKO, V. G. and YAMPOLSKI, YU. M., 1996. Ionospheric diagnostics using wave field diffraction near the caustic. Radio Sci. vol. 31, no. 5, pp. 1109-1118. DOI: 10.1029/96RS01737

13. DOLUKHANOV, M. P., 1972. Propagation of radio waves. Moscow, USSR: Svyaz Publ. (in Russian).

14. TROMSØ GEOPHYSICAL OBSERVATORY, UIT, THE ARCTIC UNIVERSITY OF NORWAY, 2015. Archive ionograms Tromsø 2003 -> today [online]. [viewed: 07.09.2018]. Available from: http://geo.phys.uit.no/iono-data/ionosonde.html

15. IZMIRAN, 2006. Moscow Digisonde DPS-4 \#045 [online]. [viewed: 07.09.2018]. Available from: http://dps.izmiran.ru/

16. IRA NASU, DEPARTMENT OF RADIOPHYSICS OF GEOSPACE, 2018. K-Indexes of geomagnetic activity [online]. [viewed: 07.09.2018]. Available from: http://geospace. com.ua/en/data/metmag_ki.php

17. TROMS $\varnothing$ GEOPHYSICAL OBSERVATORY, UIT, THE ARCTIC UNIVERSITY OF NORWAY, 2018. Geomagnetic Data [online]. [viewed: 07.09.2018]. Available from: http://flux.phys.uit.no/geomag.html

18. SPACE WEATHER PREDICTION CENTER, NATIONAL OCEANIC AND ATMOSPHERIC ADMINISTRATION, 2018. Data Service [online]. [viewed: 07.09.2018]. Available from: ftp:/ftp.swpc.noaa.gov/pub/warehouse/2013/

19. HUNSUCKER, R. D. and HARGREAVES, J. K., 2002.

The High-Latitude Ionosphere and its Effects on Radio Propagation. Cambridge: Cambridge University Press. DOI: $10.1017 / \mathrm{CBO} 9780511535758$

20. GODDARD MEDIA STUDIOS, 2013. Solar Storm Near Earth Caused by March 15, 2013 Fast CME [online]. [viewed: 07.09.2018]. Available from: https://svs. gsfc.nasa.gov/11225

\section{A. I. Reznychenko 1,2, A. V. Koloskov ${ }^{2,3}$, and Y. M. Yampolski ${ }^{2}$}

${ }^{1}$ National Technical University "Kharkiv Polytechnic Institute", 2, Kyrpychov St., 61002, Kharkiv, Ukraine

${ }^{2}$ Institute of Radio Astronomy, National Academy of Sciences of Ukraine,

4, Mystetstv St., Kharkiv, 61002, Ukraine

${ }^{3}$ National Antarctic Scientific Center of Ukraine, MES of Ukraine, 16, bul. T. Shevchenko, Kyiv, 01601, Ukraine

\section{MONITORING OF REGULAR AND SPORADIC IONOSPHERIC VARIATIONS ON THE SINGLE-HOP HF RADIO PATHS}

Purpose: Diagnostics of regular and sporadic ionospheric variations using coherent systematic monitoring of spectral characteristics of the probe HF signals on the inclined singlehop radio paths.
Design/methodology/approach: Two HF receiving stations located at the Low Frequency Observatory of the Institute of Radio Astronomy, National Academy of Sciences of Ukraine, (Martove, Kharkiv region) and in Tromsø (Norway) were recording the signals at $9.996 \mathrm{MHz}$ radiated by the radio station of the Exact Time and Frequency Service during the entire year of 2013. We estimated variations of the intensity and Doppler frequency shift (DFS) of the probe radiation derived from the power spectra recorded in the both observation points. The time series of these characteristics of the signals were compared with the independent estimates of the critical frequencies of the F2 ionospheric layer, as well as with indices of solar and magnetic activity. The correlation relationship between the time series were found.

Findings: Systematic coherent measurements of spectral characteristics of the probe HF signals on two single-hop radio paths (high- and mid-latitude) have been made. The regular (background) variations of the diurnal dependences of intensities and DFS, caused by changes under the illumination conditions of the examined radio paths during the full annual cycle of observations, have been investigated. An empirical dependence between the moments of characteristic changes in DFS, "radio-sunrise" and "radio-sunset" with the solar terminator passage at various altitudes in the midlatitude and polar ionosphere was determined. Polar day conditions and growth of absorption in the midday and afternoon play the determining role for the high-latitude radio path in summer. An index characterizing the time interval when the signals propagate below the maximum usable frequency (the duration of the "radio-day") is proposed. For the disturbed ionospheric conditions, the 27-day variations in the "radio-day" duration and the behavior of the critical frequencies of the F2 ionospheric layer are detected. The periodicity of these variations and their synchronism with the F10.7 index of solar activity can be explained by multiple influence on the ionosphere of a long-lived active region in the chromosphere during the rotation of the Sun around its own axis. The availability of using the data of HF monitoring for diagnostics of the sporadic ionospheric disturbances was demonstrated on the example of data analysis during a powerful X-ray flare on the Sun and subsequent magnetic storm.

Conclusions: The developed methods of the analysis of the data of ionospheric sounding by non-specific HF signals can be used for diagnostics and identification of ionospheric disturbances.

Key words: ionosphere, solar terminator, radio path, solar activity, magnetic storm

\section{А. І. Резниченко ${ }^{1,2}$, О. В. Колосков ${ }^{2,3}$, Ю. М. Ямпольський \\ ${ }^{1}$ Національний технічний університет “Харківський політехнічний інститут", вул. Кирпичова, 2, м. Харків, 61002, Україна \\ ${ }^{2}$ Радіоастрономічний інститут НАН України, вул. Мистецтв, 4, м. Харків, 61002, Україна \\ ${ }^{3}$ Національний антарктичний науковий центр МОН України, бул. Т. Шевченка, 16, м. Київ, 01601, Україна \\ МОНТТОРИНГ РЕГУЛЯРНИХ І СПОРАДИЧНИХ ІОНОСФЕРНИХ ВАРІАЦІЙ НА ОДНОСКАЧКОВИХ ВЧ РАДІОЛІНІЯХ}

Предмет і мета роботи: Діагностика регулярних і спорадичних іоносферних варіацій з використанням систематично- 
го когерентного моніторингу спектральних характеристик пробних ВЧ сигналів на похилих односкачкових радіолініях. Методи і методологія: Реєстрація сигналів станції Служби точного часу та частоти виконувалася на частоті $9.996 \mathrm{MГц}$ впродовж повного 2013 року в двох приймальних пунктах в Низькочастотній обсерваторії Радіоастрономічного інституту НАН України (с. Мартове, Харківської області) та в м. Тромсе (Норвегія). Реєструвалися енергетичні спектри, за якими оцінювалися варіації інтенсивності та доплерівського зміщення частоти (ДЗЧ) пробного випромінювання. Часові ряди інформаційних характеристик сигналів співставлялися з незалежними оцінками критичних частот іоносферного шару F2, а також з індексами сонячної та магнітної активності. Встановлено їх кореляційний зв'язок.

Результати: Виконано систематичні когерентні вимірювання варіацій спектральних характеристик пробних ВЧ сигналів на двох односкачкових радіолініях (високо- і середньоширотній). Протягом повного річного циклу спостережень досліджено регулярні (фонові) варіації добових залежностей інтенсивностей і ДЗЧ пробних сигналів, викликані зміною умов освітленості контрольних радіотрас. Встановлено емпіричну залежність часу характерних змін ДЗЧ і моментів “радіосходу” i “радіозаходу” з проходженням сонячного термінатора на різних висотах средньоширотної та приполярної іоносфери. Для високоширотної радіотраси влітку визначальну роль відіграють умови полярного дня і зростання поглинання в полуденний та післяполуденний час. Введено індекс, що характеризує час поширення сигналів на частоті нижче за максимально застосовну (тривалість “радіодня”). Для збурених іоносферних умов виявлені 27-денні варіації тривалості “радіодня" і поведінки критичних частот іоносферного шару F2. Періодичність цих варіацій і їх синхронізм 3 індексом сонячної активності $F 10.7$ можливо пояснити багаторазовим впливом на іоносферу активної області з тривалим часом життя в хромосфері впродовж обертання Сонця навколо своєї осі. Можливість використання даних ВЧ моніторингу для діагностики спорадичних іоносферних збурень продемонстровано на прикладі потужного рентгенівського спалаху на Сонці та магнітної бурі, що сталася після нього.

Висновок: Розроблені методики аналізу даних зондування іоносфери ВЧ сигналами неспеціального типу можуть бути застосовані для діагностики та ідентифікації іоносферних збурень.

Ключові слова: іоносфера, сонячний термінатор, радіотраса, сонячна активність, магнітна буря

Статья поступила в редакциию 25.09.2018 